Article

\title{
Automatic 2D Floorplan CAD Generation from 3D Point Clouds
}

\author{
Uuganbayar Gankhuyag (1) and Ji-Hyeong Han * \\ Department of Computer Science and Engineering, Seoul National University of Science and Technology, 232, \\ Gongneung-ro, Nowon-gu, Seoul 01811, Korea; uuganbayar@seoultech.ac.kr \\ * Correspondence: jhhan@seoultech.ac.kr; Tel.: +82-2-970-6705
}

Received: 24 March 2020; Accepted: 16 April 2020; Published: 19 April 2020

\begin{abstract}
In the architecture, engineering, and construction (AEC) industry, creating an indoor model of existing buildings has been a challenging task since the introduction of building information modeling (BIM). Because the process of BIM is primarily manual and implies a high possibility of error, the automated creation of indoor models remains an ongoing research. In this paper, we propose a fully automated method to generate $2 \mathrm{D}$ floorplan computer-aided designs (CADs) from $3 \mathrm{D}$ point clouds. The proposed method consists of two main parts. The first is to detect planes in buildings, such as walls, floors, and ceilings, from unstructured 3D point clouds and to classify them based on the Manhattan-World (MW) assumption. The second is to generate 3D BIM in the industry foundation classes (IFC) format and a 2D floorplan CAD using the proposed line-detection algorithm. We experimented the proposed method on 3D point cloud data from a university building, residential houses, and apartments and evaluated the geometric quality of a wall reconstruction. We also offer the source code for the proposed method on GitHub.
\end{abstract}

Keywords: building information modeling (BIM); 3D reconstruction; 2D floorplan CAD; 3D point clouds

\section{Introduction}

The creation of 2D floorplan computer-aided designs (CADs) of the existing buildings is a challenging task in the architecture, engineering, and construction (AEC) industry, as the as-built condition of buildings can differ from original plans due to undocumented renovations. The creation of an indoor model from existing building has been widely researched with building information modeling (BIM) and has been increasingly requested in various applications [1]. Currently, BIM processes are well established for new buildings, but most existing buildings are not maintained, refurbished, or deconstructed with BIM [2]. The advantage of the implementation of BIM in existing buildings is significant, especially for restoration, documentation, maintenance, quality control, and energy/space management.

Thus, the most important aspect is the creation of BIM without a prior model, as existing buildings usually do not have a model. To create BIM for existing buildings, 3D reconstruction is required, and a key aspect of 3D reconstruction is wall geometry modeling because it forms the basis for other elements of buildings. Currently, these objects are created manually based on large unstructured 3D point clouds acquired from the built structure. However, this process is labor-intensive and is prone to human error. Another challenge related to automated reconstruction is that $3 \mathrm{D}$ point clouds contain various types of clutter, such as furniture, and 3D point clouds lack semantic information [3].

In order to resolve these issues, we propose a fully automated method to create 2D floorplan CADs from 3D point clouds without a prior model. In the proposed method, we generate 3D reconstruction model of walls based on assumption of Manhattan-World (MW) buildings, which assumes that most 
man-made structures can be approximated by planar surfaces that are parallel to one of the three principal planes of a common orthogonal coordinate system [4].

We then generate a 2D floorplan CAD based on the 3D reconstruction of the elements. In detail, to reconstruct the wall geometry, the proposed algorithm initially detects planes from 3D point clouds and classifies them as belonging to floors, ceilings, or walls. A 2D image which contains only wall candidate points is then created and wall lines are detected. There are several line-detection algorithms, such as RANSAC [5], the Hough transform [6], and the least-square (LS) method [7]. In this paper, we propose an efficient line-detection algorithm for wall detection.

This paper is organized as follows. In Section 2, the background and related works are presented. Section 3 presents the proposed method, including the steps of data preprocessing, floor segmentation, wall detection, and 2D floorplan CAD generation. In Section 4, experimental results are discussed, including the evaluation metrics and datasets used. Finally, the concluding remarks follow in Section 5.

\section{Backgrounds and Related Works}

The main process when generating a 2D floorplan from unstructured 3D point clouds is to create BIM objects from 3D point clouds. This process can be divided into two steps, i.e., 3D reconstruction and conversion into a BIM format such as the industry foundation classes (IFC) format.

$3 \mathrm{D}$ reconstruction commonly consists of three parts after the acquisition of the point cloud: data preprocessing, plane segmentation, and plane classification. First, the 3D point cloud data are preprocessed to create structured data in order to save time during the process and achieve more efficient results. In 2D methods, the point cloud is represented as a set of images consisting of a slice of points or other information [8,9]. In 3D methods, the point cloud is restructured as a voxel octree which allows efficient neighborhood searches [10]. Subsequently, the point cloud is segmented. Typically, lines are used for point cloud segmentation in 2D methods such as RANSAC based $[8,11,12]$ and Hough transform based [13-15] methods, while planes are used for point cloud segmentation in 3D methods $[10,16,17]$. Finally, the segments are classified into categories such as floors and walls by using heuristics or machine learning techniques [18-21].

After 3D reconstruction, wall geometry modeling is the most important part of the next step. There have been several studies of the reconstruction of wall geometries. Xiong et al. and Adan and Huber reconstructed planar wall boundaries and openings based on machine learning [18,22]. Michailidis and Pajarola reconstructed severely occluded wall surfaces using Bayesian graph-cut optimization based on cell complex decomposition [23].

The relationship between elements in a building leads at the end to the BIM establishment. Additionally, element topology can serve to describe building elements. Topological data assign spatial relationship information, whereas the dimensions and locations of elements are represented by geometrical data. Types of elements and relationships with other elements are associated with these processes. Several proposed models determine topological relationships among objects automatically. Nguyen et al. presented an approach that automatically analyzes the topological relationships of building elements [24]. Belsky et al. implemented a prototype system for the semantic improvement of prior factual model files [25]. In their system, shared and slab collection concepts were acknowledged and topological, geometric, and other generic operators were used in collections of rule sets. Anagnostopoulos et al. presented a semi-automatic algorithm which calculated the boundaries and adjacency of objects in 3D point clouds [26]. It generated depictions of objects in the IFC format.

\section{Proposed Method}

The input to our method consists of unstructured 3D point clouds, and the output is the 2D floorplan CAD having topological relationships between the walls. The proposed method is composed of the $3 \mathrm{D}$ reconstruction and $2 \mathrm{D}$ floorplan CAD generation parts. In the first part, walls are detected as follows: 
1. 3D point cloud data preprocessing

2. Floor segmentation based on horizontal planes

3. Wall proposals based on vertical planes for each floor

4. Wall detection using the horizontal projection of wall proposals

5. IFC file generation from the detected wall points

In the second part, we create structural elements from the IFC file, after which, based on this information, we generate the 2D floorplan CAD. Our approach is fully automated and implemented using the PCL (point cloud library) the most popular library that works with 3D point clouds [27]. Figure 1 shows the overall flow of the proposed method. The code for the proposed method is available at https:/ / github.com/joyjo/to-generate-2D-floorplan-CAD-from-3D-point-clouds.

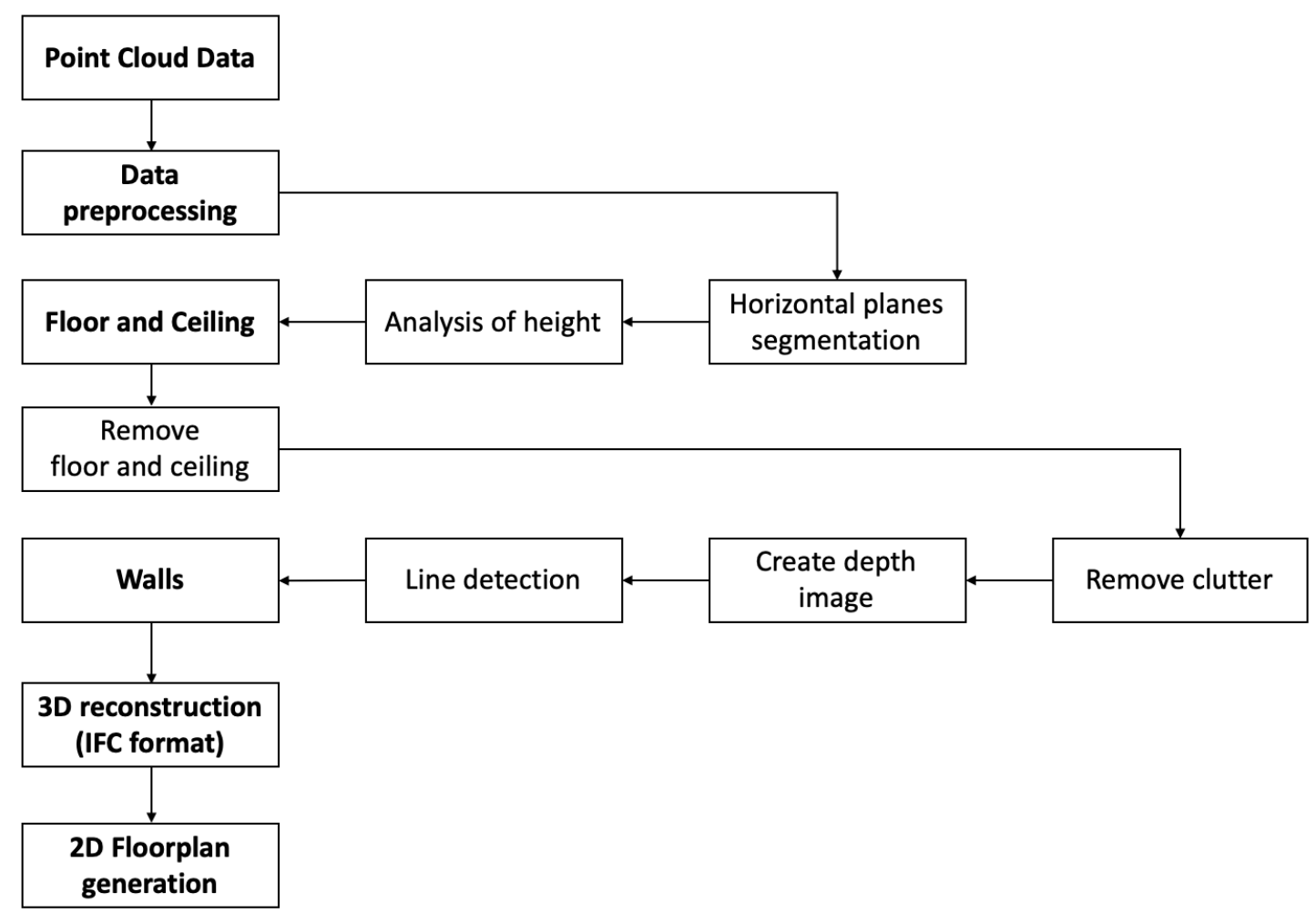

Figure 1. Overview of the proposed method that automatically creates a 2D floorplan CAD from unstructured 3D point clouds.

\subsection{Data Pre-Processing}

We used unstructured 3D point clouds as an input data from LIDAR scanners. Therefore, our algorithm requires preprocessing of the 3D point clouds in order to save time during the process and to gain more insight for meaningful element extractions, such as for floors and walls. For data preprocessing, 3D point clouds are spatially re-sampled at $1 \mathrm{~cm}$ and rotated to follow the proper orientation for a wall detection algorithm.

\subsection{Floor Segmentation}

After data preprocessing, floor segmentation is performed. We consider the assumption that floors and ceilings are horizontal and parallel to the $x-y$ plane. Therefore, the largest horizontal planes are extracted using a parallel model of the RANSAC algorithm because the floor and ceilings would contain the highest points in every possible horizontal plane. Then, detected planes are ordered by $z$-coordinate and analyzed with floor altitude threshold. Each plane is identified as a floor or a ceiling based on the altitude threshold and the 3D points of floors and ceilings are then removed to detect 
walls. The ceilings may contain clutters such as lamps, air conditioning, or ventilation openings, but these clutters are considered as a part of ceiling and removed with ceilings together.

\subsection{Wall Detection}

Detecting walls is a more complex process than detecting floors and ceilings. After removing floors and ceilings, 3D point clouds contain points belonging to walls as well as points belonging to clutter, such as furniture (e.g., desks, chairs and bookshelves). Detecting a wall is highly sensitive in cluttered data. In order to solve this problem, wall proposals are conducted by removing most of the clutter from the 3D point clouds. As shown in Figure 2, wall proposals, $W_{p}=\left\{w_{p 1}, \ldots, w_{p n}\right\}$, are calculated based on the assumption that walls are vertical and parallel to $y-z$ or $x-z$ planes. Therefore, all possible planes are extracted using a parallel model of the RANSAC algorithm. Then, our proposed algorithm retains the planes with altitudes higher than a threshold, as follows:

$$
w_{\text {pi_height }}>\text { thres } *\left(\text { Ceiling }_{\min Z}-\text { Floor }_{\max Z}\right)
$$

Here, $w_{\text {pi_height }}$ is the height of the $i$-th plane, thres is a constant value that describes the minimum plane height, Ceiling min $_{Z}$ is the minimum point of the $z$-axis for a ceiling, Floor max $_{Z}$ is the maximum point of the $z$-axis for the floor. Therefore, $\left(\right.$ Ceiling $_{\min Z}-$ Floor $_{\max Z}$ ) becomes the height of the current floor.

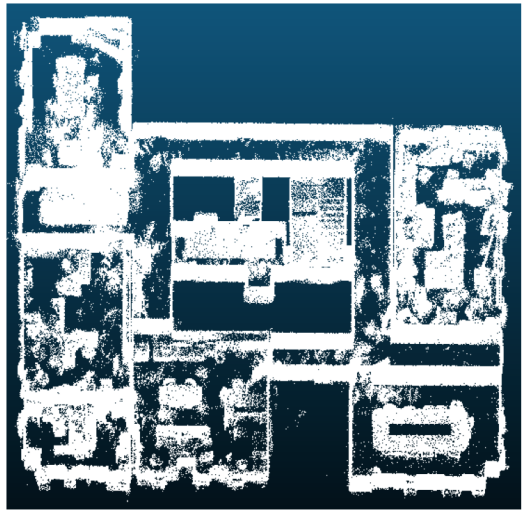

(a)

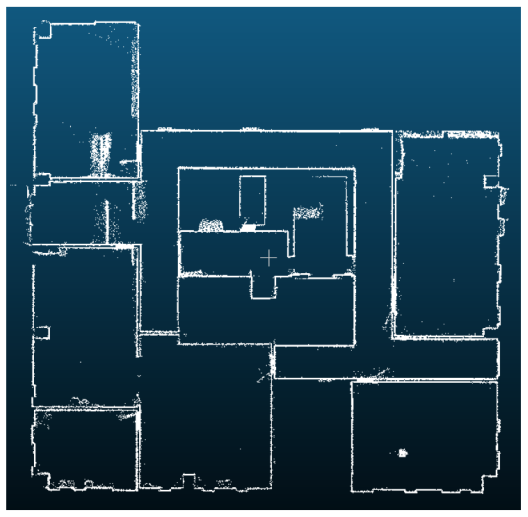

(b)

Figure 2. Result of wall proposal for seventh floor of the Robot Convergence Building at Korea University: (a) 3D point clouds after removing the floor and ceiling, and (b) 3D point clouds of wall proposal planes.

After the wall proposals, walls are detected with two steps. In the first step, we create depth image based on points of wall proposal planes that are projected onto the $x-y$ plane using Algorithm 1 . The expected result is described in Figure 3a. Algorithm 1 works as follows:

- Inputs are 3D point clouds of wall proposals and the grid size (default value for the grid size: $0.01 \mathrm{~m})$.

- $\quad 3 \mathrm{D}$ point clouds are projected into the $x-y$ plane (lines 1 and 3 ), meaning we set all $z$-axis points to zero.

- Create a grid structure from projected 3D point clouds (lines between 4 and 12). The projected $3 \mathrm{D}$ point clouds are initially sliced on the $x$-coordinate with range $[i, i+$ gridSize $]$ and then on the $y$-coordinate with range $[j, j+$ gridSize $]$, where $i$ is an $x$-coordinate value of $i$-th point and $j$ is a $y$-coordinate value of the $j$-th point. Then, the point density of each grid cell is then saved in a grid.

- Create an empty image with the height and width of the grid (line 13).

- Calculate the image intensity based on the described grid (line 14). In an image, intensity defines the pixel value; i.e., the pixel can have a value from 0 to 255 in a grayscale image. 


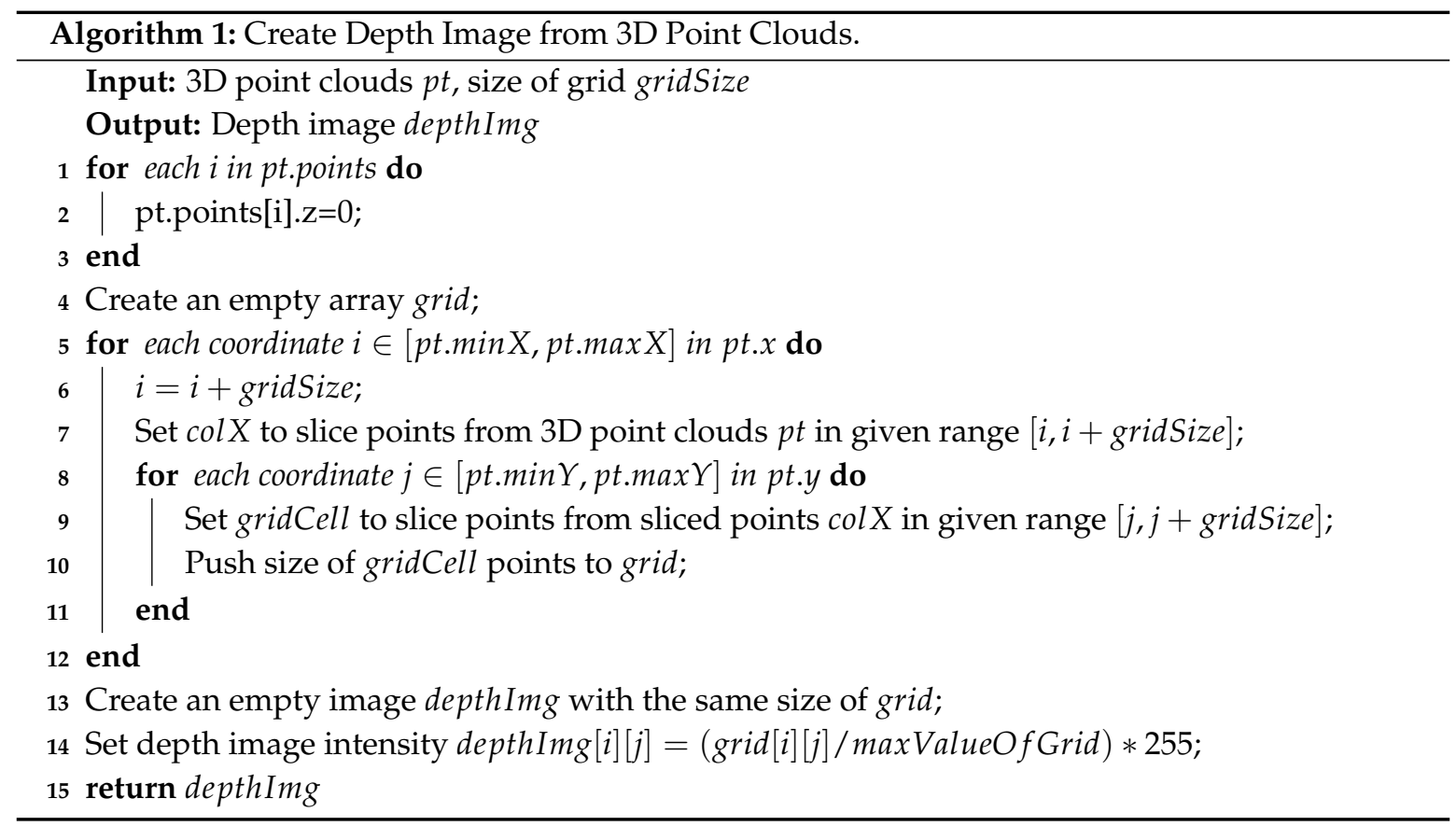

In the second step, walls are detected using the created depth image with Algorithm 2. To solve the problem of missing 3D points when the building is scanned by a LIDAR, we propose an efficient line-detection algorithm from the created depth image. The proposed algorithm finds all horizontal and vertical lines in the depth image if a line contains at least one point. All possible wall candidates are defined in each horizontal and vertical line, as presented in Figure 3b. We then detect actual walls from the wall candidate lines. The expected result is presented in Figure 3c. Algorithm 2 has the following steps:

- Inputs are the depth image that results from Algorithm 1 and the thresholds, i.e., minimum points for the wall line, minPoints; minimum length of the wall line, minLen; and determined area, detArea.

- Define the wall candidate lines, clines (lines between 1 and 6). If a pixel, depthImg $g_{i j}$, has an intensity value greater than 0 , i.e., the pixel is not black, then the pixel is a part of the wall candidate lines. Therefore, the horizontal and vertical lines that pass the pixel are drawn. In detail, the horizontal and vertical lines are described by two points: $(0, j)$ and (depthImg.cols $-1, j)$, and $(i, 0)$ and $(i$,depthImg.rows -1$)$. This process is repeatedly done for all pixels in depthImg.

- Define actual wall lines from wall candidate lines (lines between 7 and 20). First, we check the pixels in each wall candidate line, clines. If there are points around the pixel within the predefined area threshold (the default value is 50 pixels), it means that the pixel can be a part of the wall line. We set an area threshold value because there are some missing points and noise from the LIDAR sensor. We set $p$ Lines $[i]$ to 1 if the $i$-th pixel satisfies the condition, and set it to 0 otherwise. As a result, we obtain the $p$ Lines vector, which is a sequence of 0 and 1 for each wall candidate line. The size of the $p$ Lines vector is equal to the width and height of the depth image for the horizontal and vertical wall candidate lines, respectively. Based on the $p$ Lines vector, we determine the actual wall lines using the thresholds, i.e., minimum points on the wall line, minPoints, and minimum length of the wall line, minLen. For example, if pLines $=\{1,1,1,1,1,1,0,0,0,1,1,1,1,1\}$, we can see two sequences of ones. If these two sequences meet the threshold condition, they are determined to be actual wall lines. 


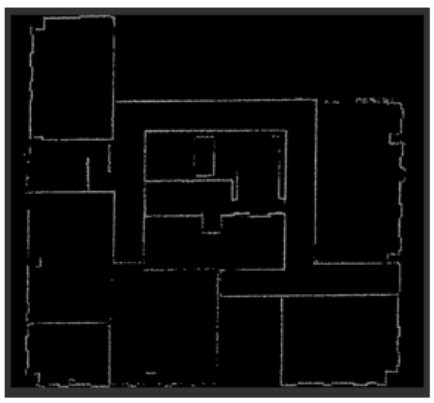

(a)

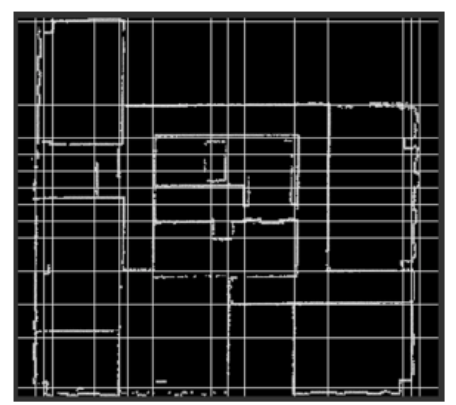

(b)

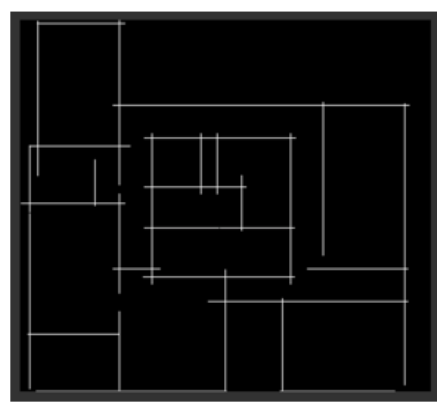

(c)

Figure 3. Result of the wall detection process for seventh floor of the Robot Convergence Building at Korea University: (a) result of the depth image, (b) results of horizontal and vertical lines, and (c) results of walls.

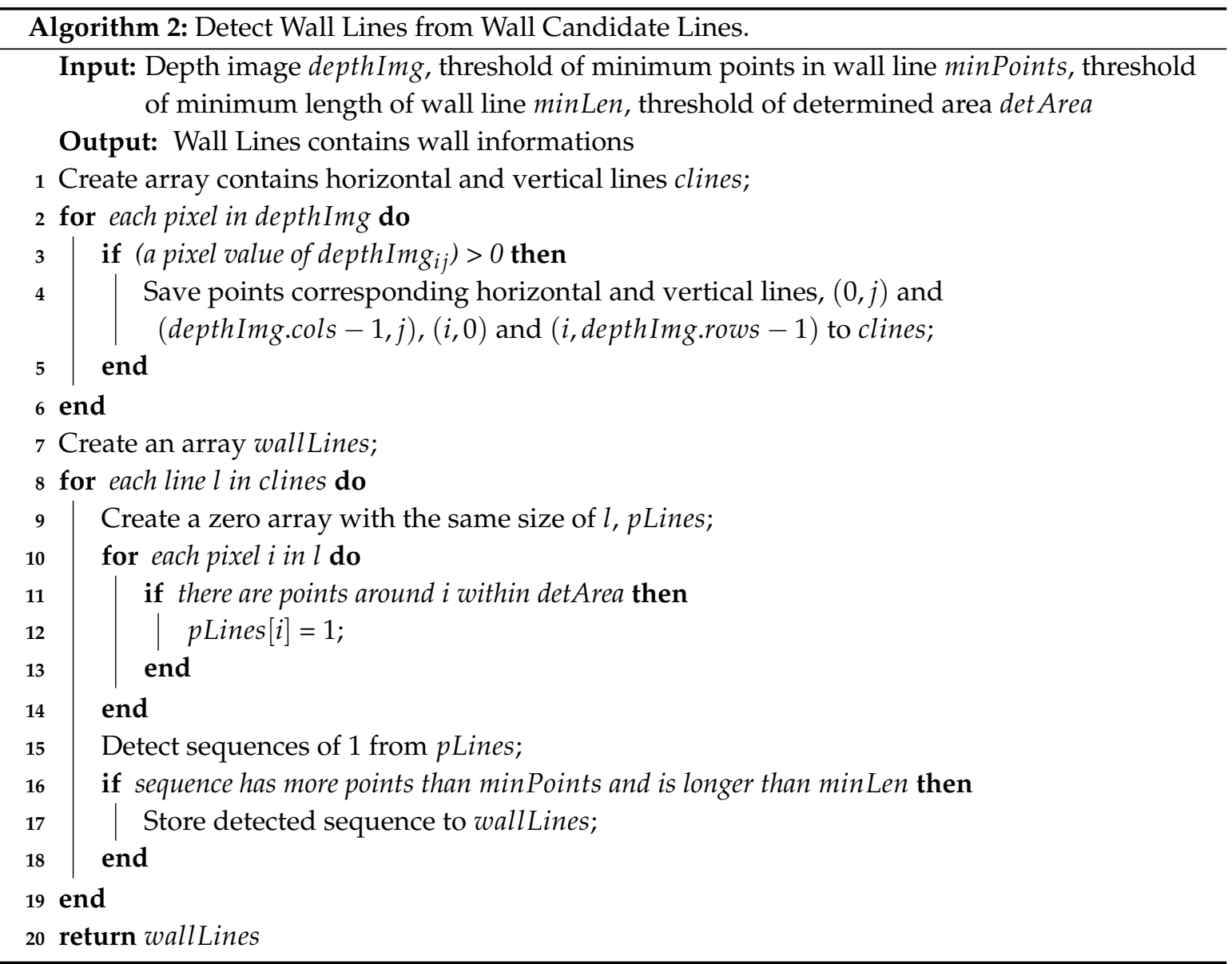

\subsection{IFC File and 2D Floorplan CAD Generation}

Using the proposed method, walls are identified successfully with information that includes the position, direction and altitudes, but they are still in the form of 3D point clouds. Therefore, 3D reconstruction of the structural elements of the building must be performed. There are two necessary steps when reconstructing walls and exporting the result into a BIM format. In the first step, we describe the 3D geometry of the elements based on information for which the wall is described as either a volume composed of eight points and six facets or as a surface composed four points and 
one facet. The second step consists of translating the 3D geometry of the elements into an IFC file. The elements describe what is expected in the building information model. A conversion phase is thus necessary to be able to integrate the results in BIM software. The IFC format is chosen as the format for the output in this approach. The IFC format is a standardized object-based file format used by the AEC industry to facilitate interoperability between building actors. Both elements and relationships between elements are described in this type of file. In order to generate a file in the IFC format from a 3D geometry of structural elements, we use the open-source library IfcOpenShell [28].

After creating the IFC format, a 2D floorplan CAD file, such as an SVG format file, is generated using the IfcConvert method in an IfcOpenShell library. The IfcConvert method converts IFC files into various output formats, which include geometrical models and which are tessellated and easy to understand.

\section{Experiments}

The proposed method was experimented on university building, residential houses, and apartment data. We evaluated the wall geometry quality using the recall and precision metric. This section explains the experimental environment and results.

\subsection{Experimental Environment}

We developed our method focused on the data of the seventh floor of the Robot Convergence Building of Korea University collected by a handheld laser scanner (ZEB-REVO). This data consists of multiple rooms with various types of clutter such as desks, chairs, and sofas as presented in Figure 4a. Therefore, we experimented on another three datasets, as presented in Figure $4 \mathrm{~b}-\mathrm{d}$, to prove that the proposed method works robustly even for unseen data which were collected by different scanners. The data in Figure $4 \mathrm{~b}$ are the second floor of the residential house collected by LIDAR (Velodyne HDL-32E), and this 3D point cloud data are very noisy and contain multiple rooms, clutters, and highly missed points in walls. The data in Figure $4 \mathrm{c}, \mathrm{d}$ are residential house and apartment which are from research that Liu et al. created a benchmark for a new vector-graphics reconstruction problem [29]. They provide 155 scans collected by Google Tango phones (Lenovo Phab 2 Pro and Asus ZenFone AR). We selected two pieces of residential data from these datasets that have multiple rooms, little clutter, and the highly missed point in walls. In this paper, we referenced these two datasets as "Tango Scan-1" and "Tango Scan-2". The details of each data are presented in Table 1. The proposed algorithm used all 3D point clouds as an input, as presented in Figure 4. In the preprocessing step, we decreased by nearly $30 \%$ of number of input point clouds and conducted structuring in voxels with a leaf size of $0.01 \mathrm{~m}$ in order to save time and retrieve more structural data.

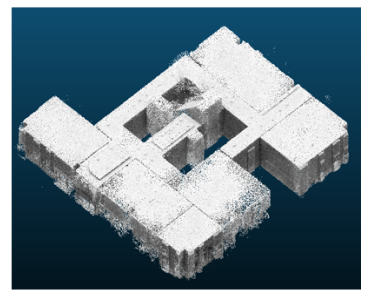

(a)

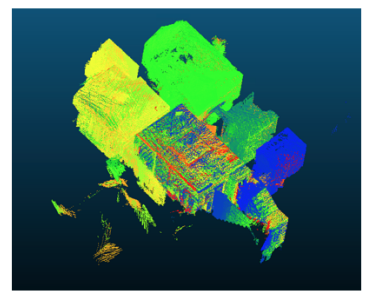

(b)

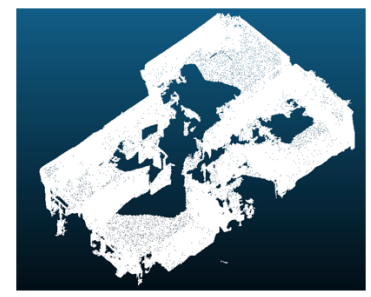

(c)

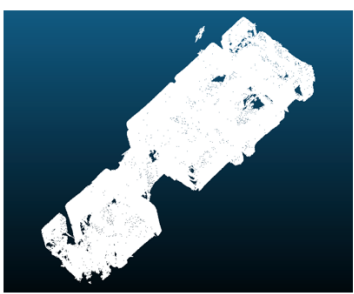

(d)

Figure 4. Input 3D point clouds: (a) seventh floor of the Robot Convergence Building at Korea University, (b) second floor of residential house , (c) Tango Scan-1 and (d) Tango Scan-2.

We evaluated the position and length of the detected walls by means of precision and recall, as used in pattern recognition, information retrieval, and classification. The main concept of these methods is the fraction of relevant instances among the retrieved instances. We computed these metrics based on the overlap between the areas of the ground truth and detected walls. We evaluated true-positive, false-positive, and false-negative cases. True-positive (TP) refers to the area of a detected 
wall that is a wall in the ground truth, false-positive $(F P)$ refers to the area of a detected wall that is not a wall in the ground truth, and false-negative $(F N)$ is the area that is a wall in the ground truth but is not detected as a wall by the proposed algorithm. Based on TP, FP, and $F N$, we calculated the precision and recall as follows:

$$
\begin{array}{r}
\text { Precision }=T P /(F P+T P) \\
\text { Recall }=T P /(F N+T P)
\end{array}
$$

Table 1. Details of datasets.

\begin{tabular}{lllllll}
\hline Dataset & Scanner & \# of Rooms & \# of Points & $\begin{array}{l}\text { Floor } \\
\text { Size }\end{array}$ & $\begin{array}{l}\text { Degree of } \\
\text { Clutter }\end{array}$ & $\begin{array}{l}\text { Degree } \\
\text { Missing Points }\end{array}$ \\
\hline $\begin{array}{l}\text { Seventh floor of the Robot } \\
\begin{array}{l}\text { Convergence Building of } \\
\text { Korea University }\end{array}\end{array}$ & ZEB-REVO & 7 & $1,969,106$ & $300 \mathrm{~m}^{2}$ & High & Low \\
\hline $\begin{array}{l}\text { Second floor of residential } \\
\text { house }\end{array}$ & $\begin{array}{l}\text { Velodyne } \\
\text { HDL-32E }\end{array}$ & 5 & $14,756,398$ & $66.6 \mathrm{~m}^{2}$ & High & High \\
\hline $\begin{array}{l}\text { Tango Scan-1 } \\
\text { phones Tango }\end{array}$ & 4 & $1,000,193$ & - & Medium High \\
\hline Tango Scan-2 & $\begin{array}{l}\text { Google Tango } \\
\text { phones }\end{array}$ & 5 & $1,000,077$ & - & Low & High \\
\hline
\end{tabular}

We defined thresholds by considering common buildings. During the wall detection process, thres $=0.9$; in Algorithm 1, gridSize $=0.01 \mathrm{~m}$; and in Algorithm 2, det Area $=50$ pixels which is identical at $50 \mathrm{~cm}$ because gridSize is $0.01 \mathrm{~m}$, minPoints $=2500$, and minLen $=100 \mathrm{~cm}$.

\subsection{Experiment Results}

During the overall wall detection process, we initially removed the floor and ceiling from the input 3D point clouds. Figure 5 shows the detected floor and ceiling point clouds of the seventh floor of the Robot Convergence Building of Korea University. Then, using Algorithms 1 and 2, we detected lines from point clouds. We proposed an efficient line-detection algorithm by considering missing 3D points and noise from sensor input. The proposed algorithm is more efficient than existing algorithms such as the faster line detection and Hough transform algorithms. Furthermore, the proposed algorithm does not require any post-processing such as the Hough transform. Figure 6 shows the comparison results of line-detection algorithms on the seventh floor of the Robot Convergence Building of Korea University and Table 2 shows the processing time for each algorithm using an Intel i7-7800X and 64 GB of memory.

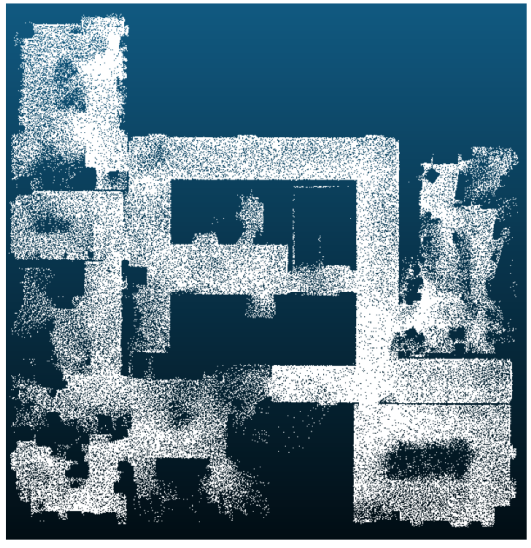

(a)

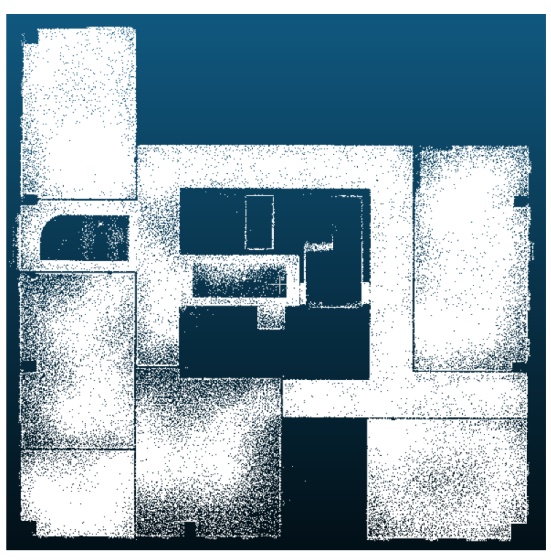

(b)

Figure 5. Result of detected 3D point clouds of the floor and ceiling: (a) floor, and (b) ceiling. Experimented on the seventh floor of the Robot Convergence Building of Korea University dataset. 


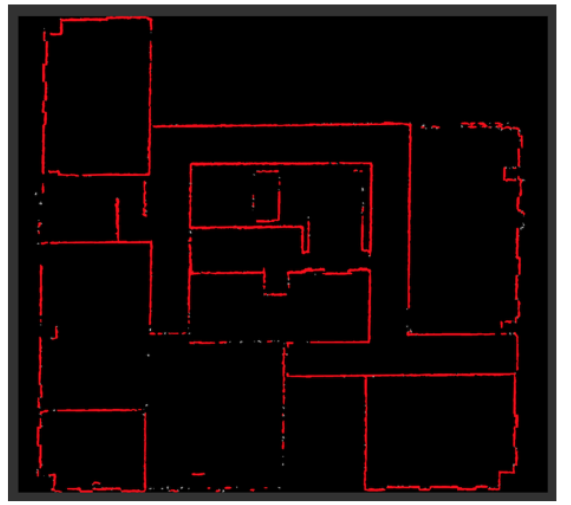

(a)

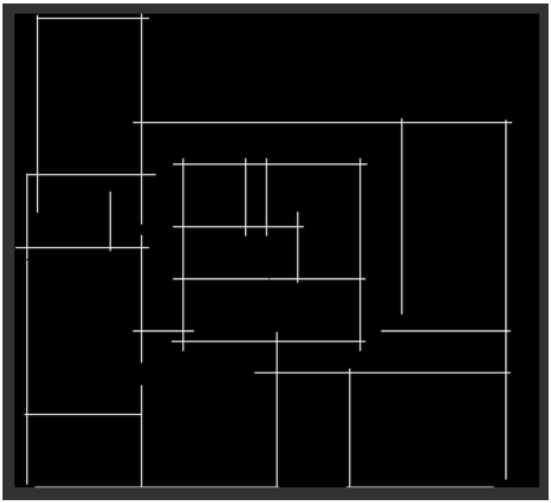

(b)

Figure 6. Comparison result of the line-detection algorithms: (a) Hough transform algorithm with post-processing, and (b) the proposed algorithm.

Table 2. Results of the processing time using the proposed algorithm and the Hough transform algorithm when detecting walls.

\begin{tabular}{cc}
\hline & Processing Time (s) \\
\hline Hough transform & 15.7166 \\
\hline Proposed algorithm & 15.6943 \\
\hline
\end{tabular}

Table 3 shows the final wall detection result. We detected $97 \%$ of the ground truth and reconstructed the detected walls in the IFC file format, as shown in Table 4.

Table 3. Wall detection evaluation results.

\begin{tabular}{clllll}
\hline & $\begin{array}{l}\text { Seventh Floor of the Robot } \\
\text { Convergence Building of } \\
\text { Korea University }\end{array}$ & $\begin{array}{l}\text { Second Floor of } \\
\text { Residential House }\end{array}$ & $\begin{array}{l}\text { Tango } \\
\text { Scan-1 }\end{array}$ & $\begin{array}{l}\text { Tango } \\
\text { Scan-2 }\end{array}$ \\
\hline TP & $92.8 \%$ & $91 \%$ & $92.2 \%$ & $97.5 \%$ \\
FT & $5.8 \%$ & $7.1 \%$ & $5.5 \%$ & $1.6 \%$ \\
FN & $2 \%$ & $2.3 \%$ & $2.2 \%$ & $0.08 \%$ \\
Precision & $94 \%$ & $92.7 \%$ & $94.3 \%$ & $98.3 \%$ \\
Recall & $97.8 \%$ & $97.5 \%$ & $97.6 \%$ & $99.1 \%$ \\
\hline
\end{tabular}

Table 4. Result of the proposed method.

\begin{tabular}{llll}
\hline Dataset & 3D Reconstruction BIM & 2D Floorplan CAD [SVG] \\
\hline $\begin{array}{c}\text { Seventh floor of the Robot } \\
\text { Convergence Building of } \\
\text { Korea University }\end{array}$ &
\end{tabular}


Table 4. Cont.

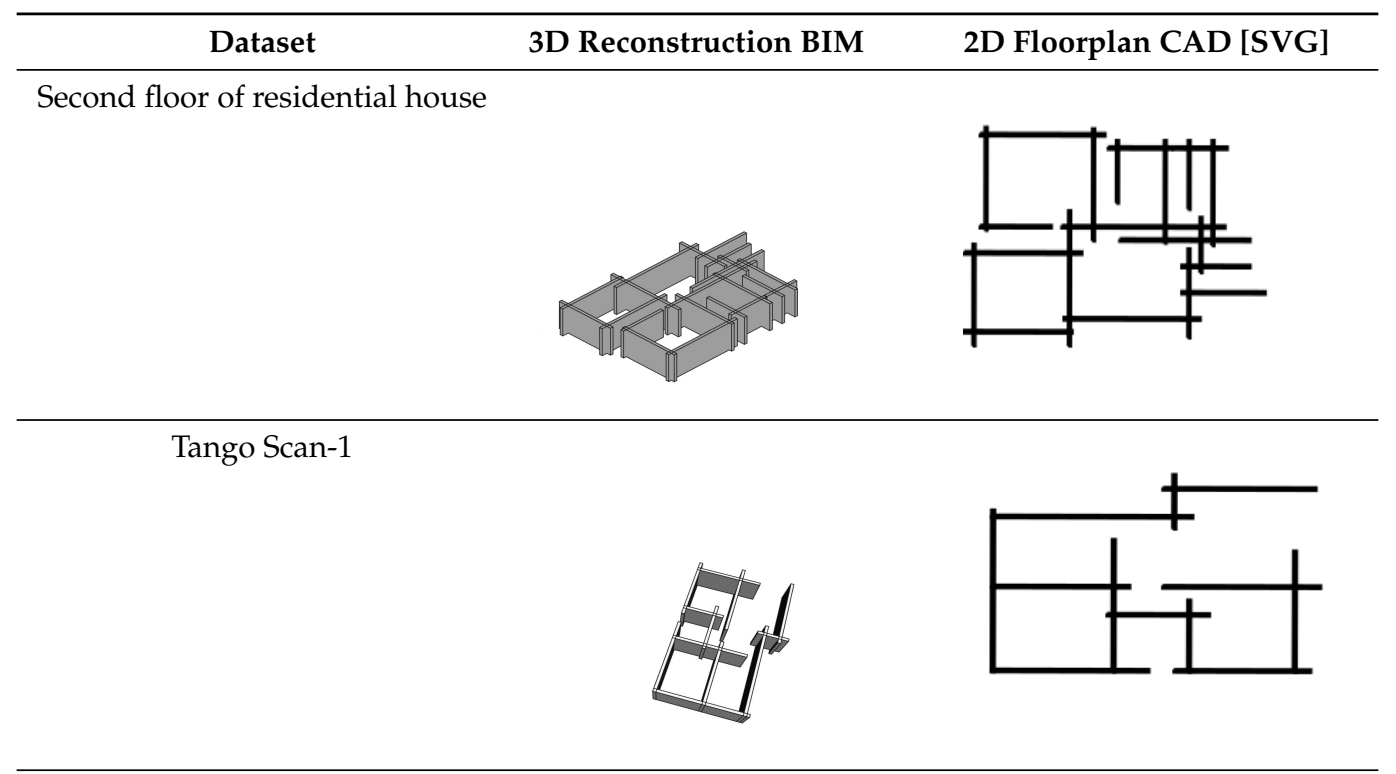

Tango Scan-2

\section{Conclusions and Future Work}

Here, we proposed a fully automated algorithm for generating 2D floorplan CADs from 3D point clouds. First, the proposed algorithm automatically detected walls. We proposed an efficient line-detection algorithm in the wall detection section. The advantages of the proposed algorithm compared to others are it does not require any post-processing and only needs certain predefined thresholds. Second, the proposed algorithm reconstructed detected walls in the BIM format, which can be edited in CAD programs. Finally, a 2D floorplan was generated using IfcOpenShell. We experimentally tested the proposed algorithm on the 3D point cloud data gathered from the seventh floor of the Robot Convergence Building of Korea University using LIDAR and second floor of residential house using LIDAR and open source residential house using Google Tango phone. More than $97 \%$ of the walls in the ground truth were detected, and the 2D floorplan was generated successfully. However, the proposed method had limitations in input data because it was more sensitive to data that have missing points in the wall. Our line detection algorithm solved this issue but still if more than approximately $55 \%$ of wall points per each wall were missed that wall was not detected as a wall line correctly. In the future, we will develop an algorithm that considers stairs and openings in buildings, such as doors and windows.

Author Contributions: Conceptualization, U.G.; methodology, U.G.; investigation, U.G.; writing-original draft preparation, U.G.; writing-review and editing, J.-H.H.; project administration, J.-H.H.; funding acquisition, J.-H.H. All authors have read and agreed to the published version of the manuscript.

Funding: This work was supported by a grant from the National Research Foundation of Korea (NRF) funded by the Korean government (MSIT) (No. 2018R1A4A1026027).

Conflicts of Interest: The authors declare no conflict of interest. 


\section{References}

1. Wang, C.; Yong, K.; Kim, C. Automatic BIM component extraction from point clouds of existing buildings for sustainability applications. Autom. Constr. 2015, 56, 1-13. [CrossRef]

2. Volk, R.; Stengel, J.; Schultmann, F. Building Information Modeling (BIM) for existing buildings-Literature review and future needs. Autom. Constr. 2014, 38, 109-127. [CrossRef]

3. Previtali, M.; Díaz-Vilariño, L.; Scaioni, M. Indoor Building Reconstruction from Occluded Point Clouds Using Graph-Cut and Ray-Tracing. Appl. Sci. 2018, 8, 1529. [CrossRef]

4. Coughlan, J.; Yuille, A. Manhattan world: Compass direction from a single image by Bayesian inference. IEEE ICCV 1999, 2, 941-947.

5. Fischler, M.A.; Bolles, R.C. Random Sample Consensus: A Paradigm for Model Fitting with Applications to Image Analysis and Automated Cartography. Commun. ACM 1981, 24, 381-395. [CrossRef]

6. Duda, R.O.; Hart, P.E. Use of the Hough Transformation to Detect Lines and Curves in Pictures. Commun. ACM 1972, 15, 11-15. [CrossRef]

7. Peter, M.; Jafri, S.; Vosselman, G. Line segmentation of $2 \mathrm{~d}$ laser scanner point clouds for indoor slam based on a range of residuals. ISPRS Ann. Photogramm. Remote Sens. Spat. Inf. Sci 2017, IV-2/W4, 363-369. [CrossRef]

8. Anagnostopoulos, I.; Patraucean, V.; Brilakis, I.; Vela, P. Detection of walls, floors and ceilings in point cloud data. In Proceedings of the Construction Research Congress 2016, San Juan, Puerto Rico, 31 May 2016.

9. Landrieu, L.; Mallet, C.; Weinmann, M. Comparison of belief propagation and graph-cut approaches for contextual classification of 3D lidar point cloud data. In Proceedings of the IGARSS2017, Fort Worth, TX, USA, 23 July 2017.

10. Vo, A.V.; Truong-Hong, L.; Laefer, D.F.; Bertolotto, M. Octree-based region growing for point cloud segmentation. ISPRS J. Photogramm. Remote Sens. 2015, 104, 88-100. [CrossRef]

11. Schnabel, R.; Wahl, R.; Klein, R. Efficient RANSAC for Point-Cloud Shape Detection. Comput. Graph. 2007, 26, 214-226. [CrossRef]

12. Ochmann, S.; Vock, R.; Wessel, R.; Tamke, M.; Klein, R. Automatic generation of structural building descriptions from 3D point cloud scans. In Proceedings of the 2014 International Conference on Computer Graphics Theory and Applications (GRAPP), Lisbon, Portugal, 5-8 January 2014; pp. 1-8.

13. Borrmann, D.; Elseberg, J.; Kai, L.; Nüchter, A. The 3D Hough Transform for plane detection in point clouds: A review and a new accumulator design. 3D Res. 2011, 2, 3. [CrossRef]

14. Okorn, B.; Xiong, X.; Akinci, B.; Huber, D. Toward Automated Modeling of Floor Plans. 2010. Available online: https://ri.cmu.edu/pub_files/2010/5/20093DPVTplanviewmodelingv13(resubmitted) .pdf (accessed on 31 December 2019)

15. Oesau, S.; Lafarge, F.; Alliez, P. Indoor scene reconstruction using feature sensitive primitive extraction and graph-cut. ISPRS J. Photogramm. Remote Sens. 2014, 90, 68-82. [CrossRef]

16. Fan, Y.; Wang, M.; Geng, N.; He, D.; Chang, J.; Zhang, J.J. A self-adaptive segmentation method for a point cloud. Vis. Comput. 2018, 34, 659-673. [CrossRef]

17. Vosselman, G.; Rottensteiner, F. Contextual segment based classification of airborne laser scanner data. ISPRS J. Photogram. Remote Sens. 2017, 128, 354-371. [CrossRef]

18. Xiong, X.; Adan, A.; Akinci, B.; Huber, D. Automatic creation of semantically rich 3D building models from laser scanner data. Autom. Constr. 2013, 31, 325-337. [CrossRef]

19. Wolf, D.; Prankl, J.; Vincze, M. Fast Semantic Segmentation of 3D Point Clouds using a Dense CRF with Learned Parameters. In Proceedings of the IEEE International Conference on Robotics and Automation (ICRA), Seattle, WA, USA, 26 May 2015.

20. Bassier, M.; Vergauwen, M.; Van Genechten, B. Automated Semantic Labelling of 3D Vector Models for Scan-to-BIM. In Proceedings of the 4th Annual International Conference on Architecture and Civil Engineering (ACE 2016), Singapore, 26 April 2016; pp. 93-100.

21. Nikoohemat, S.; Peter, M.; Oude Elberink, S.; Vosselman, G. Exploiting Indoor Mobile Laser Scanner Trajectories for Semantic Interpretation of Point Clouds. ISPRS Ann. Photogram. Remote Sens. Spat. Inf. Sci. 2017, 355-362. [CrossRef]

22. Adan, A.; Huber, D. 3D Reconstruction of Interior Wall Surfaces under Occlusion and Clutter. In Proceedings of the 2011 International Conference on 3D Imaging, Modeling, Processing, Visualization and Transmission, Hangzhou, China, 16-19 May 2011; pp. 275-281. 
23. Michailidis, G.T.; Pajarola, R. Bayesian graph-cut optimization for wall surfaces reconstruction in indoor environments. Vis. Comput. 2017, 33, 1347-1355. [CrossRef]

24. Nguyen, T.; Oloufa, A.; Nassar, K. Algorithms for automated deduction of topological information. Autom. Constr. 2005, 14, 59-70. [CrossRef]

25. Belsky, M.; Sacks, R.; Brilakis, I. Semantic Enrichment for Building Information Modeling. Comput. Aided Civ. Inf. 2016, 31, 261-274. [CrossRef]

26. Anagnostopoulos, I.; Belsky, M.; Brilakis, I. Object Boundaries and Room Detection in As-Is BIM Models from Point Cloud Data. In Proceedings of the 16th International Conference on Computing in Civil and Building Engineering, Osaka, Japan, 6-8 July 2016.

27. Rusu, R.B.; Cousins, S. 3D is here: Point Cloud Library (PCL). In Proceedings of the IEEE International Conference on Robotics and Automation (ICRA), Shanghai, China, 13 May 2011.

28. Ifcopenshell Team. The Open Source IFC Toolkit and Geometry Engine. Available online: http:/ / ifcopenshell. org/ (accessed on 31 December 2019).

29. Liu, C.; Wu, J.; Furukawa, Y. FloorNet: A Unified Framework for Floorplan Reconstruction from 3D Scans. Lect. Notes Comput. Sci. 2018, 203-219._13. [CrossRef]

(C) 2020 by the authors. Licensee MDPI, Basel, Switzerland. This article is an open access article distributed under the terms and conditions of the Creative Commons Attribution (CC BY) license (http://creativecommons.org/licenses/by/4.0/). 